\title{
The type 2 diabetes-associated variant in $T C F 7 L 2$ is associated with latent autoimmune diabetes in adult Europeans and the gene effect is modified by obesity: a meta-analysis and an individual study
}

\author{
K. Lukacs • N. Hosszufalusi • E. Dinya $\cdot$ M. Bakacs • \\ L. Madacsy $\cdot$ P. Panczel
}

Received: 15 June 2011 / Accepted: 28 October 2011 / Published online: 23 November 2011

(C) Springer-Verlag 2011

\begin{abstract}
Aims/hypothesis The variants of transcription factor 7-like 2 (TCF7L2) gene have been proposed to be associated with latent autoimmune diabetes in adults (LADA). We sought to confirm the possible association in Europeans and to examine the interaction between one gene variant and clinical data.

Methods The TCF7L2 rs7903146 C-to-T polymorphism was genotyped in 211 LADA, 1,297 type 2 diabetic, 545 type 1 diabetic and 1,497 control individuals from Hungary. A meta-analysis of our and previously published studies was performed to evaluate the size and the heterogeneity of the gene effect.

Results The meta-analysis yielded a significant effect of TCF7L2 T allele (OR 1.28; $p<0.0001$ ) on LADA risk without heterogeneity among Europeans. The $\mathrm{T}$ allele conferred equally strong susceptibility to LADA and type 2 diabetes. In the Hungarian dataset, the T allele was associated with LADA and type 2 diabetes, but not with type 1 diabetes.
\end{abstract}

K. Lukacs $(\bowtie) \cdot$ L. Madacsy

1st Department of Paediatrics, Semmelweis University,

Bókay u 53-54,

1083 Budapest, Hungary

e-mail: krisztinalukacs@hotmail.com

K. Lukacs $\cdot$ N. Hosszufalusi $\cdot$ P. Panczel

3rd Department of Internal Medicine, Semmelweis University,

Budapest, Hungary

E. Dinya

Institute of Health Informatics, Semmelweis University,

Budapest, Hungary

M. Bakacs

Pharmaproject-Statistics Incorporation,

Budapest, Hungary
T allele carriers had significantly lower BMI than patients with the CC genotype in the LADA and type 2 diabetes groups ( $p=0.0021$ and $p=0.0013$, respectively). In both diseases, the diabetes risk was significantly higher in the non-overweight than in the overweight BMI category ( $p=0.0013$ and $p<0.0001$, respectively); susceptibility to LADA was increased by 2.84 -fold in non-overweight individuals compared with overweight ones.

Conclusions/interpretation The meta-analysis demonstrates that TCF7L2 rs7903146 polymorphism is a populationindependent susceptibility locus for LADA in Europeans. The effect size is similar for LADA and type 2 diabetes. The gene effect on diabetes risk may be modulated by BMI, such that the lower the BMI, the higher the gene effect.

Keywords BMI - Genetic susceptibility - LADA · Meta-analysis $\cdot T C F 7 L 2 \cdot$ Type 2 diabetes
Abbreviations
LADA Latent autoimmune diabetes in adults
PAR Population-attributable risk
TCF4 Transcription factor 4

\section{Introduction}

The WHO lists latent autoimmune diabetes in adults (LADA) as a slowly progressing subgroup of autoimmunity-mediated type 1 diabetes [1]. However, LADA shows significant differences from this type, e.g. less serious symptoms at onset and slow progression towards insulin dependence, which are usually associated with type 2 diabetes [2].

According to a new theory, diabetes occurs on a continuous spectrum from type 1 through LADA to type 
2 diabetes, with entities not clearly separable because of overlap in the pathomechanisms [3]. In 2008, Cervin and colleagues [4] first proposed that LADA is genetically an admixture of type 1 and type 2 diabetes after they had found an association between LADA and transcription factor 7-like 2 (TCF7L2) gene (10q25.3), a variant (rs7903146 C-to-T polymorphism) of which is the most common susceptibility locus for type 2 diabetes.

The aim of our study was to assess the contribution of the TCF7L2 rs7903146 polymorphism to LADA risk in patients of European ancestry. This TCF7L2 variant was therefore genotyped in patients with LADA, type 2 diabetes and type 1 diabetes, as well as in controls, all from Hungary, and a meta-analysis was performed combining our dataset with data published previously [4-7]. The heterogeneity of gene effect between LADA and type 2 diabetes was also assessed. In the Hungarian dataset, the possible interaction between the polymorphism and age at onset, sex, BMI and residual beta cell function was also examined.

\section{Methods}

Participants The case-control study included 211 LADA (mean \pm SD: 112 men/99 women, age at diagnosis $44.94 \pm$ 8.26 years, BMI $25.12 \pm 4.62 \mathrm{~kg} / \mathrm{m}^{2}$ ), 1,297 type 2 diabetic (636 men/661 women, age at diagnosis $52.63 \pm 9.85$ years, BMI $29.52 \pm 4.95 \mathrm{~kg} / \mathrm{m}^{2}$ ) and 545 type 1 diabetic (291 boys/ 254 girls, age at diagnosis $8.11 \pm 4.08$ years, BMI $16.06 \pm$ $3.67 \mathrm{~kg} / \mathrm{m}^{2}$ ) patients. All cases were of European ancestry, diagnosed and/or followed up at Semmelweis University, Budapest, Hungary. The 1,497 healthy controls ( 765 men/732 women, age $37.62 \pm 10.92$ years, BMI $26.35 \pm 4.46 \mathrm{~kg} / \mathrm{m}^{2}$ ) had normal fasting glucose levels and no family history of diabetes. Informed consent was obtained from all individuals; the study was approved by the national Ethics Committee and conducted according to the principles of the Declaration of Helsinki.

Classification of diabetes LADA was diagnosed if: (1) the age at onset was $>35$ years; (2) autoantibody-positivity was documented for islet cell, GAD, insulinoma-associated protein 2 or insulin antibodies; and (3) insulin treatment was not started in the first 6 months after diagnosis. Type 2 diabetic individuals were $>35$ years, antibody-negative and insulin-independent at diagnosis. Type 1 diabetic patients were $<15$ years, had autoantibody-positivity and required prompt insulin therapy at diagnosis.

Data collection BMI data were divided into two subgroups according to classification by the WHO, i.e. nonoverweight (BMI $<25 \mathrm{~kg} / \mathrm{m}^{2}$ or BMI less than $1 \mathrm{SD}$ above the mean) and overweight (BMI $\geq 25 \mathrm{~kg} / \mathrm{m}^{2}$ or BMI $1 \mathrm{SD}$ or more above the mean). Fasting serum C-peptide concentrations were measured by radioimmunoassay (normal value $0.35-1.15 \mathrm{nmol} / \mathrm{l}$ ) at disease onset without glucose-lowering treatment.

Genotyping All participants were genotyped for the TCF7L2 rs7903146 polymorphism using an allelic discrimination assay (TaqMan; Applied Biosystems, Foster City, CA, USA). No deviation from Hardy-Weinberg equilibrium was observed in controls and the totality of the patient groups.

Statistical analysis Disease association was calculated by a logistic regression model adjusted for age, sex and BMI. Group comparisons were carried out by analysis of covariance, followed by Tukey's test as post-hoc analysis. Categorical variables were compared using Pearson's $\chi^{2}$ test. The Breslow-Day test was used to assess allelic heterogeneity.

Meta-analysis was performed using MIX software [8]. Between-study heterogeneity assessment by Cochran's Q-test was not statistically significant. Thus, the MantelHaenszel fixed-effect method was performed to pool the allelic OR. The population attributable risk (PAR) was calculated according to the method of Tong [9]. All $p$ values were two-tailed and considered significant at $p<0.05$. Each analysis was performed using SPSS for Windows (version 17.0; SPSS, Chicago, IL, USA).

\section{Results}

In the Hungarian dataset, the rs7903146 polymorphism showed significant disease associations with LADA (T allelic OR 1.34, 95\% CI 1.07, 1.68; $p=0.0127$ ) and type 2 diabetes (OR 1.49, 95\% CI 1.13, 1.73; $p<0.0001$ ), but not with type 1 diabetes (OR $0.98,95 \%$ CI $0.73,1.31 ; p=0.5219$ ). The associations were age- and sex-independent. The PAR of the T allele was $15.21 \%$ in the LADA and $16.96 \%$ in the type 2 diabetes patient groups.

The meta-analysis of published studies and our results comprised a total of 999 LADA patients and 5,358 control individuals of European ancestry, and yielded a modest, but significant effect for the TCF7L2 rs 7903146 T allele on LADA risk (OR 1.28, 95\% CI 1.15, $1.42 ; p<0.0001 ;$ Fig. 1). Between-study heterogeneity was not significant $(p=0.9288)$. The PAR of the T allele was $12.99 \%$. The disease-associated $\mathrm{T}$ allele frequencies showed a north-south geographic gradient in patient and background populations. Comparison of the effect size of the TCF7L2 gene variant rs7903146 C-to-T polymorphism among 999 LADA and 4,529 type 2 diabetic patients revealed homogeneity $(p=0.2423)$. 


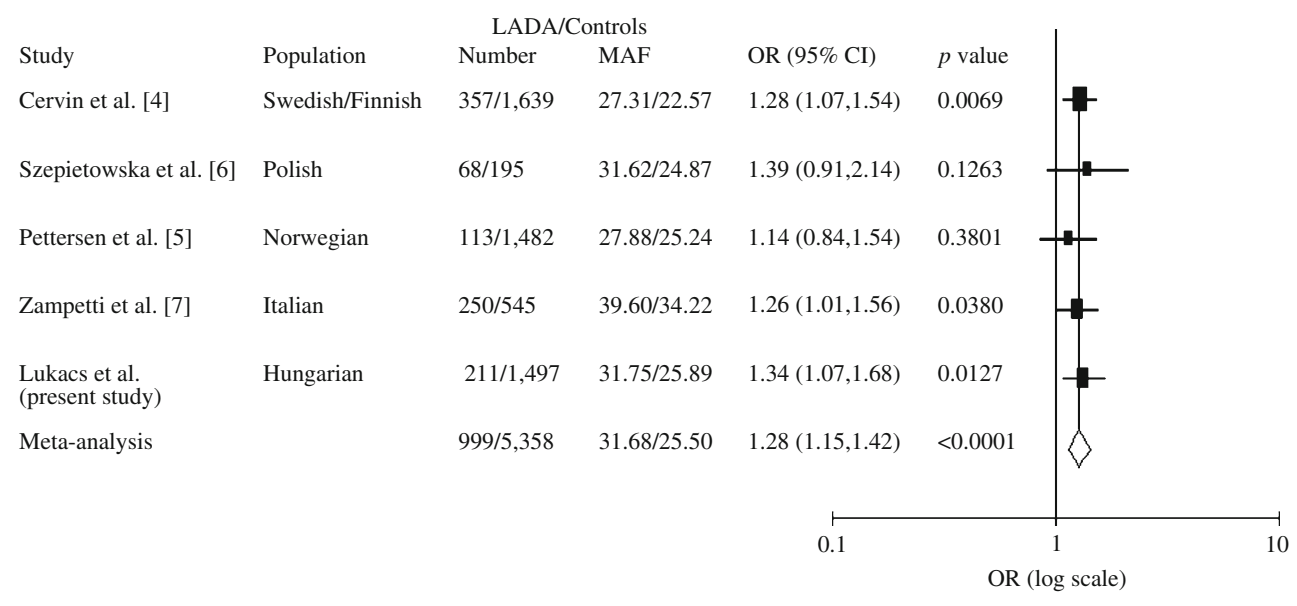

Fig. 1 Association of the TCF7L2 rs7903146 polymorphism with LADA in European populations. Data are shown for allelic ORs based on allele counts. Six populations of European ancestry from distinct geographical locations were included in the meta-analysis. No

In the Hungarian dataset, $\mathrm{T}$ allele carriers had significantly lower mean BMI values than patients with the CC genotype in the LADA (mean \pm SD: $24.29 \pm 2.74$ vs $\left.26.26 \pm 2.82 \mathrm{~kg} / \mathrm{m}^{2}, p=0.0021\right)$ and type 2 diabetic $\left(29.10 \pm 5.06\right.$ vs $\left.30.04 \pm 4.77 \mathrm{~kg} / \mathrm{m}^{2}, p=0.0013\right)$ groups. A similar interaction was not seen in controls and type 1 diabetes (data not shown). We further analysed the effect of this $T C F 7 L 2$ variant on diabetes risk in non-overweight vs overweight BMI categories (Table 1). T allele carrying was associated with LADA only in the non-overweight group (OR 1.61, 95\% CI 1.21, 2.12; $p=0.0011$ ); polymorphism-related susceptibility to diabetes was increased by 2.84 -fold in non-overweight compared with overweight heterogeneity was observed across studies (Cochran's Q test $p=$ $0.9288)$. Studies are ordered by publication date. ORs and $95 \%$ CIs are shown. The size of OR symbols is proportionate to the study sample size. MAF (\%), minor allele frequency

LADA patients $(p=0.0013)$. In type 2 diabetes, $\mathrm{T}$ allele carriers showed an increased risk compared with controls, both in the non-overweight (OR 1.93, 95\% CI 1.49, $2.50 ; p<0.0001)$ and in the overweight (OR $1.36,95 \% \mathrm{CI}$ $1.14,1.63 ; p=0.0038)$ categories; polymorphism-related susceptibility was higher in non-overweight than in overweight individuals $(p<0.0001)$.

$\mathrm{T}$ allele carriers among LADA patients tended to have lower fasting serum C-peptide levels (mean $\pm \mathrm{SD}, \mathrm{CT} / \mathrm{TT}$ genotype $0.24 \pm 0.18 \mathrm{nmol} / 1$ vs CC genotype $0.29 \pm$ $0.23 \mathrm{nmol} / 1, p=0.0841)$. This difference was significant in type 2 diabetic individuals $(0.81 \pm 0.23$ vs $0.87 \pm 0.24 \mathrm{nmol} / \mathrm{l}$, respectively, $p=0.0352$ ).

Table 1 Analysis of the associations between TCF7L2 rs7903146 polymorphism and LADA, type 2 and type 1 diabetes by cases vs controls and BMI categories

\begin{tabular}{|c|c|c|c|c|c|c|c|c|}
\hline \multirow[t]{2}{*}{ Cases by BMI status ${ }^{\mathrm{a}}$} & \multicolumn{3}{|c|}{ Genotype $(n)$} & \multicolumn{5}{|l|}{$\mathrm{T}$ allele } \\
\hline & $\mathrm{CC}$ & $\mathrm{CT}$ & $\mathrm{TT}$ & Frequency $(\%)$ & $\begin{array}{l}\text { Diabetes vs control, } \\
\text { OR }(95 \% \mathrm{CI})\end{array}$ & $p$ value & $\begin{array}{l}\text { Per BMI status, } \\
\text { OR }(95 \% \mathrm{CI})\end{array}$ & $p$ value \\
\hline \multicolumn{9}{|l|}{ Non-overweight } \\
\hline Control & 375 & 270 & 43 & 25.9 & - & - & - & - \\
\hline Type 2 diabetes & 71 & 136 & 34 & 42.3 & $1.93(1.49,2.50)$ & $<0.0001$ & $2.26(1.68,3.07)$ & $<0.0001$ \\
\hline LADA & 56 & 72 & 16 & 36.1 & $1.61(1.21,2.12)$ & 0.0011 & $2.84(1.55,5.21)$ & 0.0013 \\
\hline Type 1 diabetes & 238 & 166 & 35 & 26.9 & $1.05(0.87,1.28)$ & NS & $1.05(0.68,1.62)$ & NS \\
\hline \multicolumn{9}{|l|}{ Overweight } \\
\hline Control & 445 & 309 & 55 & 25.9 & - & - & - & - \\
\hline Type 2 diabetes & 514 & 446 & 96 & 30.2 & $1.36(1.14,1.63)$ & 0.0038 & 1.00 (reference) & - \\
\hline LADA & 43 & 18 & 6 & 22.4 & $0.86(0.56,1.31)$ & NS & 1.00 (reference) & - \\
\hline Type 1 diabetes & 57 & 41 & 8 & 26.9 & $1.07(0.77,1.48)$ & NS & 1.00 (reference) & - \\
\hline
\end{tabular}

Allelic ORs for diabetes were calculated by logistic regression model adjusted for age and sex

${ }^{\mathrm{a}} \mathrm{BMI}$ status is based on the International Classification and Child Growth Standards of the WHO: non-overweight BMI $<25 \mathrm{~kg} / \mathrm{m}^{2}$ for adults and $<1$ SD above mean for children; overweight $\mathrm{BMI} \geq 25 \mathrm{~kg} / \mathrm{m}^{2}$ for adults and $1 \mathrm{SD}$ or more above mean for children 


\section{Discussion}

Previous studies have shown that type 1 diabetes susceptibility genes (HLA family, PTPN22 and INS) increase the risk of $\operatorname{LADA}[4,5]$, thereby suggesting that LADA represents a subgroup of autoimmunity-mediated type 1 diabetes. However, there is strong evidence that LADA differs from type 1 diabetes in the pathomechanisms involved [10]. Recent association studies have raised the possibility that type 2 diabetes susceptibility genes contribute to risk of LADA. TCF7L2 is the most strongly associated type 2 diabetes susceptibility gene [9], but has not been previously associated with type 1 diabetes [11].

This study was conducted to prove the role of nonautoimmune mechanisms in the pathogenesis of LADA by confirming the independent association between TCF7L2 gene and LADA in European populations.

Our results show an association between the TCF7L2 rs7903146 C-to-T polymorphism and LADA in a Central European population. In the Hungarian dataset, the diseaseassociated $\mathrm{T}$ allele increased susceptibility to LADA by 1.34-fold, which corresponds to a 15\% PAR. We confirmed that TCF7L2 is not associated with type 1 diabetes.

We performed a meta-analysis of previously published studies [4-7] in addition to our data. It provided convincing statistical evidence for a role of TCF7L2 rs7903146 polymorphism in LADA risk. A north-south geographic gradient was seen in the frequency of the disease-associated $\mathrm{T}$ allele, both in LADA and control populations. Despite these substantial differences in risk allele frequencies, the effect size of TCF7L2 gene on LADA risk was very similar across the populations studied. The absence of betweenstudy heterogeneity may indicate that the effect of TCF7L2 gene is population-independent among Europeans. In addition, a comparable magnitude of gene effect was observed between LADA and type 2 diabetic populations.

In the Hungarian study, we demonstrated for the first time in LADA that the TCF7L2 variant rs7903146 increased diabetes risk in non-overweight patients only. Similarly to the data published recently by Cauchi et al. [12], the gene effect was also significantly stronger in type 2 diabetic patients with lower BMI. No association was found in type 1 diabetic patients, regardless of BMI categories. These findings suggest a phenotype $\times$ genotype interaction in LADA and type 2 diabetes, with the TCF7L2 gene effect on diabetes risk possibly being modulated by BMI, such that the lower the BMI, the higher the gene effect. Further investigations are necessary to confirm this possible interaction.

Similarly to Szepietowska's observation [6], we also found a tendency in $\mathrm{T}$ allele carriers with LADA to have lower fasting serum C-peptide levels. This interaction was significant in our type 2 diabetic group. The TCF7L2 gene encodes a transcription factor, transcription factor 4 (TCF4), and the TCF4-catenin complex increases secretion of glucagon-like peptide 1 , which is involved in beta cell proliferation and insulin secretion. One putative effect of TCF7L2 gene may be a further decrease of insulin secretion via non-autoimmunity-mediated beta cell dysfunction.

In summary, our meta-analysis from six European populations shows strong evidence that the TCF7L2 variant rs7903146 C-to-T polymorphism contributes to susceptibility to LADA. The effect size of this TCF7L2 gene variant is similar in LADA and type 2 diabetes. Its effect on diabetes risk may be modulated by BMI status in both diseases, such that the lower the BMI, the higher the gene effect.

Acknowledgements This study was supported by the Hungarian Diabetes Association. The technical assistance of A. Szigeti and support from the Hungarian National Blood Service are gratefully acknowledged. We are most grateful to all patients and control individuals who volunteered to participate in our study.

Contribution statement $\mathrm{KL}, \mathrm{NH}, \mathrm{LM}$ and $\mathrm{PP}$ contributed to conception and design, and manuscript drafting and revision. ED and $\mathrm{MB}$ contributed to analysis and interpretation of data and manuscript revision. All authors have approved the final version of manuscript to be published.

Duality of interest The authors declare that there is no duality of interest associated with this manuscript.

\section{References}

1. Alberti KG, Zimmet PZ (1998) Definition, diagnosis and classification of diabetes mellitus and its complications. Part 1: diagnosis and classification of diabetes mellitus provisional report of a WHO consultation. Diabet Med 15:539-553

2. Hosszufalusi N, Vatay A, Rajczy K et al (2003) Similar genetic features and different islet cell autoantibody pattern of latent autoimmune diabetes in adults (LADA) compared with adultonset type 1 diabetes with rapid progression. Diabetes Care 26:452-457

3. Brooks-Worrell B, Palmer JP (2011) Is diabetes mellitus a continuous spectrum? Clin Chem 57:158-161

4. Cervin C, Lyssenko V, Bakhtadze E et al (2008) Genetic similarities between latent autoimmune diabetes in adults, type 1 diabetes, and type 2 diabetes. Diabetes 57:1433-1437

5. Pettersen E, Skorpen F, Kvaloy K, Midthjell K, Grill V (2010) Genetic heterogeneity in latent autoimmune diabetes is linked to various degrees of autoimmune activity: results from the Nord-Trondelag Health Study. Diabetes 59:302-310

6. Szepietowska B, Moczulski D, Wawrusiewicz-Kurylonek N, Grzeszczak W, Gorska M, Szelachowska M (2010) Transcription factor 7-like 2-gene polymorphism is related to fasting $\mathrm{C}$ peptide in latent autoimmune diabetes in adults (LADA). Acta Diabetol 47:83-86

7. Zampetti S, Spoletini M, Petrone A et al (2010) Association of TCF7L2 gene variants with low GAD autoantibody titre in LADA subjects (NIRAD Study 5). Diabet Med 27:701-704 
8. Bax L, Yu LM, Ikeda N, Tsuruta H, Moons KG (2006) Development and validation of MIX: comprehensive free software for meta-analysis of causal research data. BMC Med Res Methodol 6:50

9. Tong Y, Lin Y, Zhang Y, Yang J, Liu H, Zhang B (2009) Association between TCF7L2 gene polymorphisms and susceptibility to type 2 diabetes mellitus: a large Human Genome Epidemiology (HuGE) review and meta-analysis. BMC Med Genet 10:15
10. Naik RG, Brooks-Worrell BM, Palmer JP (2009) Latent autoimmune diabetes in adults. J Clin Endocrinol Metab 94:4635-4644

11. Field SF, Howson JM, Smyth DJ, Walker NM, Dunger DB, Todd JA (2007) Analysis of the type 2 diabetes gene, TCF7L2, in 13,795 type 1 diabetes cases and control subjects. Diabetologia $50: 212-213$

12. Cauchi S, Nead KT, Choquet H et al (2008) The genetic susceptibility to type 2 diabetes may be modulated by obesity status: implications for association studies. BMC Med Genet 9:45 